\title{
Development of a novel high straining backward extrusion process
}

\author{
Qiang Wang ${ }^{1, *}$, Zhimin Zhang ${ }^{1}$, Xubin $\mathrm{Li}^{1}$, and Huifang Zhang ${ }^{1}$ \\ ${ }^{1}$ College of Materials Science and Engineering, North University of China, 030051 Taiyuan, China
}

\begin{abstract}
In this study, a new method of backward extrusion is proposed. In this new process, a punch with a movable mandrel was designed. A hollow billet was firstly backward extruded and subsequently upset with the use of the punch after the mandrel returned. The extrusion and upsetting processes were successively executed in order for a higher effective strain to be imposed and a fibrous tissue flow direction to be controlled. In order for the capability of this process to be investigated, experimental and finite element (FE) methods were used. The effective strain of the final part prepared by both the conventional and the new process were compared along the bottom radial and wall axial direction respectively. In the results, it is shown that the plastic strain applied through the processed sample was approximately higher in twice the value of the sample processed via conventional backward extrusion. Consequently, this may improve the mechanical properties and anisotropy of the final products. The difference of the UTS and the TYS between radial and tangential at the bottom was less than $3 \%$. This new process has proven to be promising for parts with a central hole at the bottom production in order for the parts low performance to be improved.
\end{abstract}

Keywords. Extrusion, Strain, Aluminium

\section{Introduction}

Nowadays, the extrusion process has a great important role in manufacturing industries. Between various conventional deformation processing methods, the backward extrusion (BE) method has become one of the most promising manufacturing processes in usage, due to its material savings and high surface quality $[1,2]$. Moreover, backward extrusion has been used in steel, aluminum and magnesium alloy forming for the production of hollow-shape symmetric and cylindrical products [3]. Due to the evolution of the microstructures and mechanical properties of the deformed materials being directly related to the amount of plastic deformation, the phenomenon associated with strain development must be understood [4]. This is critical, considering the fact that most existing backward extrusion procedures suffer from a smaller deformation percentage of the imposed effective strain within the processed parts $[5,6]$. This may consequently lead to an unfavorable microstructure inhomogeneity and poor overall mechanical performance of the products [7].

Recently, extensive studies have been performed for various new techniques, based on the extrusion process, for imposing a higher effective strain and a better strain homogeneity. The conventional direct extrusion process with a high ratio in one/two steps [8, 9] and the hydrostatic extrusion with an extra-high ratio [10] were studied. The multiple direct extrusion (MDE) process was earlier proposed [11] as a new technique for grain refinement. A repetitive indirect extrusion technique, known as accumulative back extrusion (ABE), was introduced as a novel extrusion technique for the first time in 2009[12]. ABE is a kind of SPD process in which back extrusion (BE) and compression are cyclically executed. Certain studies are presented regarding this method [13-17]. A novel cyclic forwardbackward extrusion (CFBE) method, consisting of the forward-backward extrusion followed by constrained back-pressing, was earlier proposed for UFG materials $[18,19]$ production. A new backward extrusion utilizing a small diameter billet was also recently described as a technique for grain refinement [20]. A novel low temperature indirect extrusion process utilizing artificial cooling was devised, and the effects of artificial cooling during indirect extrusion on the microstructure and tensile properties of an extruded commercial AZ31 alloy were studied [21].

In this study, a new backward extrusion method for a hollow billet using the punch with the movable mandrel to be produced, named Expanding-Reducing Extrusion (ERE), including conventional indirect extrusion followed by upsetting, is proposed. The description of this novel high-straining process, emphasizing the effective strain, metal flow behavior and the experimental results for evaluation of the potential of both grain refinement and mechanical properties improvement, is presented. 


\section{Principle of the new backward extrusion process}

Schematic illustrations of the conventional and new backward extrusion methods are shown in Figs. 1 and 2. During the conventional backward extrusion process, the solid billet was initially placed in the matrix and combined with the pressure of the punch, the material was compressed and flow was initiated into the gap between the punch and the matrix. The principles of the new backward extrusion process are shown in Fig. 2 where the equipment used, contained an innovative punch with a movable mandrel. The billet, in the form of a hollow cylinder, was placed into the die cavity (Fig. 2a). Following the mandrel insertion into the billet, the billet was pressed by the punch in order for the material to be back-extruded into the gap, between the punch and the die (Fig. 2b). Upon the return of the mandrel, the bottom of the extruded part was upset with the use of the same punch for the diameter to be reduced and the fibrous tissue flow direction (Fig. 2c) to be controlled.

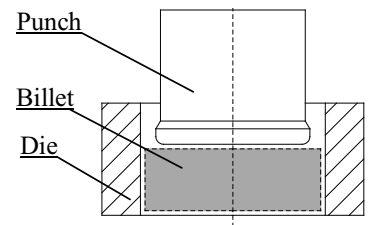

(a)

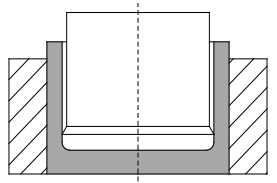

(b)
Fig.1. Schematic view of the conventional backward extrusion

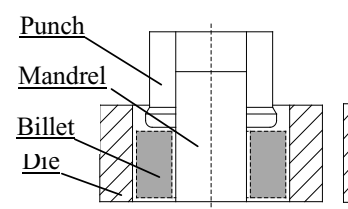

(a)

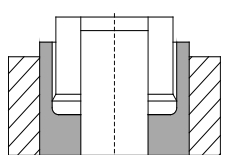

(b)

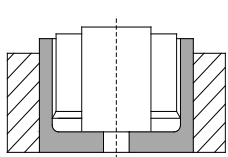

(c)
Fig.2. Schematic view of the new method of backward extrusion

\section{Experimental and FE procedure}

\subsection{Simulation}

The DEFORM 3D-6.1 software was used for FE simulation. The employed material in this study was 7A04 aluminum alloy (Al-6.15wt $\% \mathrm{Zn}-2.63 \mathrm{wt} \% \mathrm{Mg}-$ $1.6 \mathrm{wt} \% \mathrm{Cu})$. The size of the billet and mold was predetermined as shown in table 1 . The true stress-strain data of the 7A04 alloy, based on the results of the compression test, were inserted into the FE software. The material model was defined as elastic-plastic. All die parts were considered as rigid bodies. The billet was defined as a deformable object during analysis. A Tetrahedral element type was used, and the mesh sensitivity test was executed. The suitable mesh number was reported to be 50000 . The selected friction type was the shear friction type, and due to the nature of the process, the friction factor $(\mathrm{m})$ was selected to be 0.3 . The simulation type was the Lagrangian Incremental.
Global re-meshing was chosen, and the type of interference depth selected was relative and its value selected to be 0.7 . The conjugate-Gradient was chosen as the solver of the simulation.

\subsection{Experimental procedure}

The dimensions of all objects in the experiment matched the dimensions of the simulated objects perfectly, in order for the results of the simulation to be validated. The deformation processing was executed in the temperature of $470^{\circ} \mathrm{C}$, whereas the temperature error was controlled within a divergence of ${ }^{\circ} \mathbb{E 5}$ The forming mold was pre-heated to a matching temperature with the billet. In order for the friction between aluminum specimens and tools to be reduced, the contact surfaces were lubricated beforehand with oil-based graphite. The equipment utilized was a hydraulic oil press machine of $30 \mathrm{MN}$ in capacity and the average forming speed of a ram was $10 \mathrm{~mm} / \mathrm{s}$.

The products processed were solution treated at $470^{\circ} \mathrm{C}$ for $2 \mathrm{~h}$ and then subsequently aged at $125^{\circ} \mathrm{C}$ in an air furnace. Specimens were cut from the bottom and wall and machined into tensile specimens of $5 \mathrm{~mm}$ gauge diameter and $25 \mathrm{~mm}$ gauge length. Tensile testing was carried out on an Instron-10kN material test machine at speed of $1 \mathrm{~mm} / \mathrm{min}$. Microstructure examination was performed in an optical microscope (OM). The grain size was determined using a linear intercept method from a large number of non-overlapping measurements.

Table 1. Computational condition in finite element (unit: $\mathrm{mm}$ )

\begin{tabular}{|c|c|c|c|c|c|}
\hline & \multicolumn{3}{|c|}{ size } & \multicolumn{2}{c|}{ Billet size } \\
\cline { 2 - 6 } & I.D & O.D & $\begin{array}{c}\text { Hole } \\
\text { diameter }\end{array}$ & Diameter & $\begin{array}{c}\text { Lengt } \\
\mathrm{h}\end{array}$ \\
\hline $\begin{array}{c}\text { Conventional } \\
\text { method }\end{array}$ & 485 & 515 & 0 & 510 & 80 \\
\hline New method & 485 & 515 & 280 & $\begin{array}{c}\text { O.D510/ } \\
\text { I.D400 }\end{array}$ & 190 \\
\hline
\end{tabular}

\section{Results and discussion}

\subsection{Equivalent strain analysis}

The effective strain distribution of the samples, prepared by both the conventional and the novel method of backward extrusion, are shown in Fig.3, respectively. The observed effective strain contours display the difference between the effective strain of the traditional and the new backward extrusion to be very high. Furthermore it can be observed from Fig.3, that the equivalent strain of the sample prepared by traditional backward extrusion from the solid billet is extremely low, particularly at the bottom of the component. While relative to employing expanding-reducing extrusion from the hollow billet, which makes the deformations become large clearly. The equivalent strain of the bottom and the wall was increased. This was interpreted that the deformation increased with the use of the novel method of backward extrusion, instead of the conventional method of backward extrusion. 


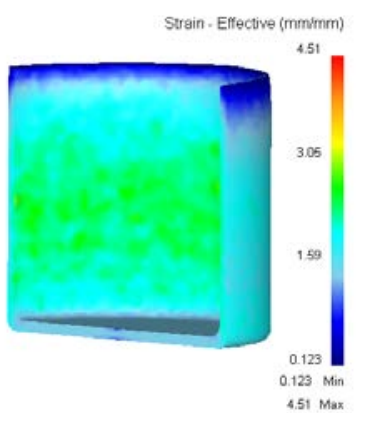

(a)

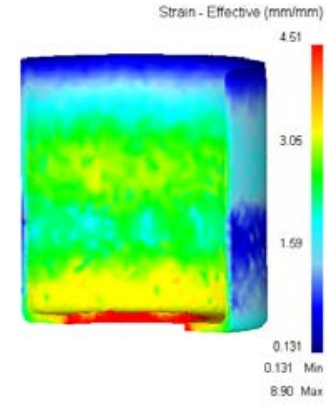

(b)
Fig. 3. Distribution of effective strain for the sample processed by (a) conventional and (b) new backward extrusion

The effective strain value of 11 points was selected to be used for the bottom length in the middle node of thickness, whereas the 11 points location was the same in both methods and the distance for each point application method was $10 \mathrm{~mm}$ from the center hole surface to the exterior area, respectively. Shown in Fig.4 , are the quantitative values of the effective strain through the bottom length of the produced part, by both described processes. Clearly observed from the graph, is the effective strain obtained from the bottom length of the part produced from the novel method of backward extrusion, having at least twice the value of the effective strain obtained from any point of the part manufactured from the traditional method of backward extrusion, although the variation range of the effective strain was relatively high. Spreading smoothly towards the part interior, the effective strain in the case of the novel backward extrusion process was gradually increased in significance. The effective strain in the novel process was even higher, reaching approximately three times the strain of the part produced from the conventional method.

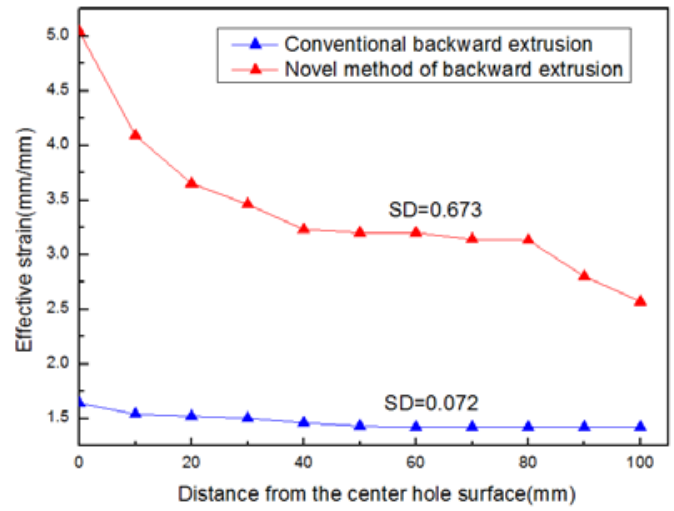

Fig. 4. Comparison of effective strains along the bottom length of processed products

The quantitative values of the effective strain along the wall length of the produced parts by both methods are compared, as shown in Fig.5. The effective strain value of 12 points was selected to be used along the wall length in the middle node of thickness, whereas the application location of the 12 points value was the same in both methods and the distance of each two points application was $30 \mathrm{~mm}$ from the bottom to the top of the part. The Standard Deviation value of effective strain for the conventional and the novel method of backward extrusion was 0.3 and 0.28 respectively. It was interpreted that the homogeneous degree of effective strain in the novel backward extrusion is relatively higher. The effective strain of the novel method of backward extrusion through the wall length of the part was higher by 1.5 times the effective strain at any point of the parts having been manufactured by the traditional method of backward extrusion.

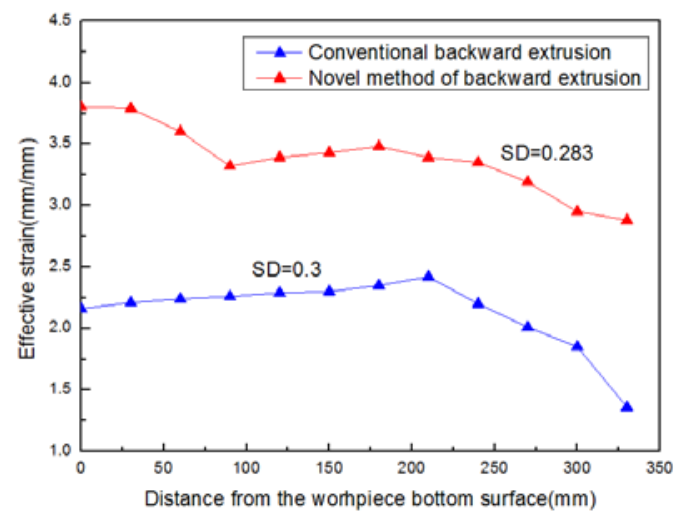

Fig. 5. Comparison of effective strains along the wall length of processed products

\subsection{Microstructure and mechanical properties}

The 7A04 alloy cylinder parts were extruded successfully without cracks or discontinuities according to the present technique. The prepared initial hollow billet and the extruded and upset processed sample are both shown in Fig. 6(a, b and c), accordingly.

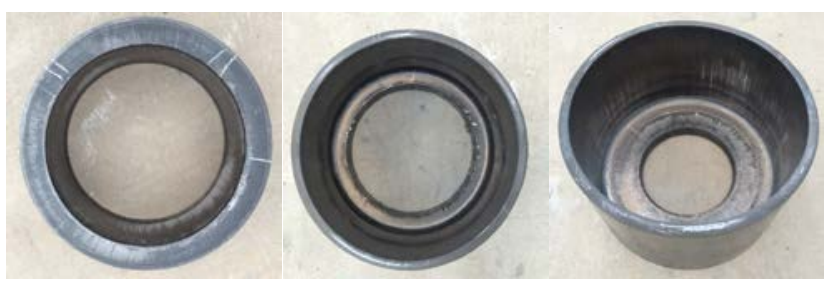

Fig.6. (a) Initial hollow billet, (b) Extrusion sample, (c) Extrusion sample with upsetting

Fig. 7 presents the mechanical properties of $7 \mathrm{~A} 04$ alloy part extruded by new process at different positions and directions. The UTS and the TYS were over $560 \mathrm{MPa}$ and $480 \mathrm{MPa}$ respectively, the elongation was above $10 \%$ in both bottom and wall positions. The mechanical properties reach a peak at wall axial direction, but the difference from the bottom was very small. The difference of the UTS and the TYS between radial and tangential in the bottom was less than $3 \%$. It is noted that the mechanical properties anisotropy was obviously reduced due to the uniformity of deformation. For comparative purposes, the mechanical properties of 7A04 alloy part extruded by conventional process also were tested. The UTS and the TYS were about $540 \mathrm{MPa}$ and $460 \mathrm{MPa}$ respectively, the elongation was about $8 \%$. It can be seen that mechanical properties were 
remarkably improved for the 7A04 alloy component when it is extruded by the new process.

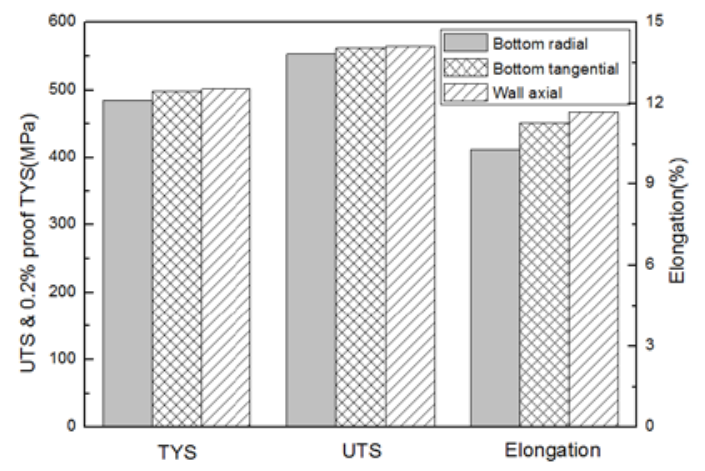

Fig.7. Mechanical properties of 7A04 alloy part obtained by new method of backward extrusion

Fig. 8 shows the microstructure of the transverse sections of the extruded part of 7A04 alloy in T6 condition. It is obvious that the extrusion specimens have recrystallized and the grains refine greatly. The calculation shows that the average grain size $(7.1 \mu \mathrm{m})$ of wall specimen was less than that $(7.5 \mu \mathrm{m})$ of bottom specimen. It is obvious that grain refinement by dynamic recrystallization is very effective in alloy. At the same time, a large amount of fine and uniformly distributed second phase particles can be observed both bottom and wall positions.

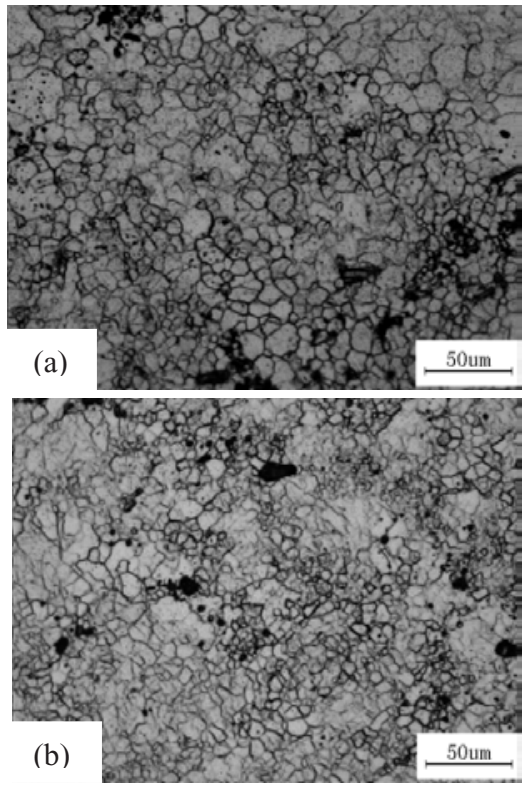

Fig.8. Microstructure of 7A04 alloy part extruded in (a) bottom and (b) wall position

The novel method of backward extrusion can apply a higher plastic strain compared to the strain of the traditional method, especially at the bottom of the component. A large body of researchers observed that the microstructure and formation of small sized grains corresponded to effective strain [22] amount. A higher plastic strain causes to the grain size of the microstructure to decrease and consequently enhance mechanical properties [23]. Therefore, this new method proves to be promising for the production of cylinder parts with higher strength compared to parts produced from the conventional technique.

\section{Conclusions}

In this study, the traditional and new backward extrusion processes were simulated for the 7A04 aluminum alloy products, with the use of the DEFORM3D numerical simulation software. The effective strain diagram for both kinds of forming processes was analyzed and the effective strain values of the product are compared. The results showed that the novel method of backward extrusion can impose a higher effective strain. The effective strain of the workpieces in the novel method of backward extrusion were at least 1.5 times in value compared to the corresponding workpieces of the conventional processing method that reached 2 times in value, the strain at the bottom of the workpieces. Correspondingly, the grain of the final products was finer and the mechanical properties of the products improved. This new expanding-reducing extrusion (ERE) method proved to be extremely promising for higher strength parts production comparing to the conventional extrusion method.

The authors are grateful for the research support of the Program for the Top Young and Middle-aged Innovative Talents of Higher Learning Institutions of Shanxi.

\section{References}

1. D.Hinz, A.Kirchner, D.Brown, B.M.Ma, O. Gutfleisch, J. Mater. Process. Tech 135, 358-365 (2003)

2. M.M.Moshksar, R.Ebrahimi, Int. J. Mech. Sci 40, 1247-1263 (1998)

3. B.Barišić, Z.Car, M.Ikonić, METABK 47, 313-316 (2008)

4. Hyoung Seop Kim,Min Hong Seo,Sun lg Hong, J. Mater. Process. Tech 113, 622-626 (2001)

5. Roberto B.Figueiredo, Paulo R.Cetlin,Terence G.Langdon, Acta. Mater 55, 4769-4779 (2007)

6. Megumi Kawasaki, Roberto B.Figueiredo,Terence G.Langdon, J. Mater. Sci 47, 7719-7725( 2012)

7. F.P.E.Dunne, Int J Plasticity 14,413-433 (1998)

8. H.K. Lin,J.C.Huang, Mater.Trans 43, 2424-2432 (2002)

9. Yongjun Chen, Qu Dong Wang, Jianguo Peng, Chun Quan Zhai, Mater. Sci. Forum 503-504, 865870 (2006)

10. Y. M. Hwang, M. T. Yang, Key. Eng. Mater 233,311-316 (2003)

11. L.Zaharia,R.Chelariu,R.Comaneci, Mater.Sci.Eng.A 55, 293-299 (2012)

12. S.M. Fatemi-Varzaneh, A. Zarei-Hanzaki, Mater. Sci. Eng. A 504,104-106 (2009)

13. H. Alihosseinia, G. Faraji, A.F. Dizaji, K. Dehghani, Mater. Charact 68,14-21(2012) 
14. G.Faraji, H.Jafarzadeh, H.J.Jeong, M.M.Mashhadi, H.S.Kim, Mater. Design 35, 251-258 (2012)

15. N. Haghdadi, A. Zarei-Hanzaki, D. Abou-Ras, M.H. Maghsoudi, A. Ghorbani, M. Kawasaki, Mater. Sci .Eng. A 595, 179-187 (2014)

16. S.M.Fatemi-Varzaneh, A.Zarei-Hanzaki, S.Izadi, J. Mater. Sci 46,1937-1944 (2010)

17. G.Faraji, M.M.Mashhadi, H.S.Kim, Mater. Sci. Eng. A 528, 4312-4317(2011)

18. H.Alihosseini, M.AsleZaeem, K.Dehghani, Mater. Lett 68, 204-208(2012)

19. H. Alihosseini, M. Asle Zaeem, K. Dehghani, H.A. Shivaee, Mater. Lett 74, 147-150(2012)

20. V. Shatermashhadi, B. Manafi, K. Abrinia, G. Faraji, M. Sanei, Mater. Design 62, 361-366 (2014)

21. Beomcheol Kim, Chan Ho Park, Ha Sik Kim, Bong Sun You and Sung Soo Park, Scripta. Mater 76, 2124 (2014)

22. G.Faraji, A.Babaei, M.M.Mashhadi, K.Abrinia, Mater. Lett 77, 82-86 (2012)

23. S.M.Fatemi-Varzaneh, A.Zarei-Hanzaki, Mater. Sci. Eng. A 504, 104-109 (2009) 